\title{
Heat Flux Distribution Estimation for CSP Applications
}

\author{
Konstantinos G. Stokos (corresponding author) ${ }^{1 凶}$, Evgeny V. Votyakov ${ }^{1}$, and Costas N. \\ Papanicolas ${ }^{1}$
}

${ }^{1}$ Energy Environment and Water Research Center, The Cyprus Institute, 2121 Nicosia, Cyprus

${ }$ k.stokos@cyi.ac.cy

\begin{abstract}
In this paper the concept of a new method for the estimation of the heat flux distribution and the total power in CSP applications is presented. This method requires appropriate analysis of the temperature evolution on a target, or directly on a receiver. A 3-D thermal conduction model with boundary conditions to take into account the convection and radiation losses has been developed. A parametric analysis was performed and we checked how the physical parameters affect the applicability of the method. Having proven numerically the potential of this method, it was experimentally implemented in the central tower CSP plant of The Cyprus Institute at PROTEAS facilities successfully. The experience gained from the numerical and experimental application of this method is discussed.
\end{abstract}

Keywords: Concentrated Solar Power, Heat Flux Distribution, Renewable Energy

\section{Introduction}

The knowledge of the heat flux distribution developed on the receiver of each Concentrated Solar Power (CSP) plant is necessary. The heat flux distribution will be used as a feedback for the heliostats aiming control strategy and its spatial integration will give the heat power input to the receiver, which is useful for its efficient operation.

As it is stated by Roger et al. [1] several methods have been presented in the literature for the measurement of the heat flux, appropriate for different receiver types and showing different characteristics, which could be classified in three categories; direct, indirect and measurement-supported simulation methods.

Direct methods use flux sensors which directly measure the heat flux in the position they are placed. Then solar flux contours are created using interpolation techniques. In $1981 \mathrm{King}$ and Arvizu [2] used a stationary bar instrumented with 64 circular foil heat flux gauges that a heliostat beam could be swept over.
The system required a 20 s time period for the measurement necessitating the water cooling of the bar, and the measurement inaccuracy was estimated at $\pm 10 \%$. Kodama et al. [3] measured the solar flux distribution on a beam-down solar concentrating system in Miyazaki moving an array of thirteen Gardon gauges. The linearity of the sensors showed to be within $2 \%$ and the response time was less than $250 \mathrm{~ms}$.

The calibration of the heat flux sensors is a critical point and at present there is not a standard procedure for their calibration. Ballesterin et al. 2004 [4] and Ballesterin et al. 2006 [5] applied a new calorimetric approach for the calibration of the Gardon gauges reducing the uncertainty in the calibration constant from 3\% to less than $2 \%$. In 2014 Guillot et al. [6] published a work on a comparison of calibration approaches presented from different laboratories applied on 4 sensors; 3 Gardon heat flux gauges and a water calorimeter. An agreement within $10 \%$ of all the sensors was observed, with the radiometers requiring slightly less than $1 \mathrm{~s}$ and the calorimeter requiring about $30 \mathrm{~s}$ as a response time.

Indirect methods use cameras and often calibration and scaling with flux gauges or calorimeters is required. In 2002 Ulmer et al. [7] presented a flux mapping system developed for the EURODISH concentrating dish at PSA. A water-cooled Lambertian target was placed in the beam path and a CCD-camera was taking images of the focal spot. The calibration was achieved from the relation of the integrated gray value and the calculated total power. In 2012 Ho and Khalsa [8] presented a novel indirect method (PHLUX) for the estimation of the heat flux distribution without using any sensors for calibration. The only additional information is the direct normal irradiance and the reflectivity of the receiver. A relative error of about $2 \%$ was found on a Lambertian surface using this method. Lee et al. [9] measured the heat flux in a diffuse/Lambertian target using a CCD camera and the heat flux gage for calibration. The purpose was to evaluate the optical performance of a solar furnace and then they compared the obtained flux maps with 
a ray tracing code. Ferriere et al. [10] presented a similar indirect method for the flux measurement in Themis solar tower in France. The measurement was done on a moving target which was placed in front of the receiver.

Measurement supported simulation methods use raytracing tools for the heat flux estimation. They seem to be easier and more flexible and they provide high resolution heat flux maps. However, validation of these methods is required through either direct or indirect systems regularly. In 2016 Ebert et al. [11] presented a new measurement-supported simulation approach for the estimation of the solar input power. The uncertainty of the solar input was estimated to be from $-1.3 \%$ to $+6.3 \%$ which contributed to the overall uncertainty in the SOLUGAS receiver to be between $2.8 \%$ and $+7.7 \%$.

In this paper the concept of a new method for the heat flux measurement, which could be classified in the direct methods, is numerically demonstrated. This method is based on the appropriate analysis of the temperature evolution over time when a heliostat or more heliostats are aiming on a target or directly on a receiver. In the beginning the theory behind this methodology is presented, then a numerical model to check how the physical parameters affect the applicability of the methodology and finally experimental data for the heat flux measurement on a target, which is placed above the iSTORE receiver in PROTEAS facilities in Cyprus [12], is also given. After the gained numerical and experimental experience, issues related to the proposed methodology are discussed.

\section{Methodology - Energy Balan- ce}

Energy balance imposes that when one or more heliostats focus on a target or receiver the power entering each finite volume is equal to the power exiting the finite volume plus the heat stored in the finite volume which increases its temperature.

$$
Q_{e n t}=Q_{e x t}+d m \cdot C_{p} \cdot \frac{d T}{d t}
$$

The power entering the finite volume is either by neighboring finite volumes through conduction either by the sunlight and the power exiting the finite volumes is either by conduction to the neighboring finite volumes or by convection and radiation to the environment. If thermal equilibrium is assumed before the sunlight reflection, there is not conductive power from or to the neighboring finite volumes and convective/radiative losses to the environment. When sunlight is reflected onto the target the temperature of each finite volume starts to increase and we can assume that in the beginning $(t=0)$ the temperature increase is only because of the power from the sunlight.

$$
a \cdot Q_{s}=\left.d m \cdot C_{p} \cdot \frac{d T}{d t}\right|_{t=0}
$$

$a$ is the absorption coefficient of the target, $Q_{s}$ is the reflected power on the front surface of the finite volume, $d m$ is the mass of the finite volume, $C_{p}$ is the heat capacity of the material of the target and $\left.\frac{d T}{d t}\right|_{t=0}$ is the temperature time derivative in the beginning. Dividing by the front surface area $d A_{s}$ for each control volume we have

$$
a \cdot q_{s}=\left.\frac{d m}{d A_{s}} \cdot C_{p} \cdot \frac{d T}{d t}\right|_{t=0}
$$

where $q_{s}$ is the reflected heat flux. Integrating for the whole target the total power on the target can be given by the following equation.

$$
a \cdot Q_{s}^{t o t}=\left.m \cdot C_{p} \cdot \frac{d T_{a v g}}{d t}\right|_{t=0}
$$

where $Q_{s}^{t o t}$ is the total power reflected onto the target and $T_{a v g}$ is the average temperature of the target. We see that either for the measurement of the heat flux on each finite volume (equation 3 ) or for the measurement of the total power from the sun on the target (equation 4) we need the accurate measurement of the temperature time derivative, either for the finite volume or for the whole target respectively. Then the proposed methodology focuses on the accurate measurement of these temperature time derivatives.

\section{Thermal conduction model}

In order to study the applicability of the methodology and perform a parametric analysis to check how the independent parameters affect it a thermal conduction model has been developed. It is a 3-D transient model for structured numerical meshes without volumetric heat sources, developed to analyze the temperature evolution on a flat target for simplicity reasons (figure 1 ) when one or more heliostats aim on it. The model is expressed by the time dependent diffusion equation 5 :

$$
\frac{d T}{d t}=\frac{k_{t}}{\rho \cdot C_{p}}\left(\frac{d^{2} T}{d x^{2}}+\frac{d^{2} T}{d y^{2}}+\frac{d^{2} T}{d z^{2}}\right)
$$

where constants $k_{t}, \rho$ and $C_{p}$ are the thermal conductivity, density and the specific heat at constant pressure of the target. Equation 5 can be discretized applying appropriate spatial and temporal discretization techniques. The temperature second spatial derivatives were numerically approximated using the following 
finite difference formulas:

$$
\begin{gathered}
\frac{d^{2} T}{d x^{2}}=\frac{T_{i+1}-2 T_{i}+T_{i-1}}{\Delta x_{i}^{2}} \\
\frac{d^{2} T}{d y^{2}}=\frac{T_{j+1}-2 T_{j}+T_{j-1}}{\Delta y_{j}^{2}} \\
\frac{d^{2} T}{d z^{2}}= \\
\frac{2 \Delta z_{k-1} T_{k+1}-2\left(\Delta z_{k-1}+\Delta z_{k}\right) T_{k}+2 \Delta z_{k} T_{k-1}}{\Delta z_{k}^{2} \Delta z_{k-1}+\Delta z_{k-1}^{2} \Delta z_{k}}
\end{gathered}
$$

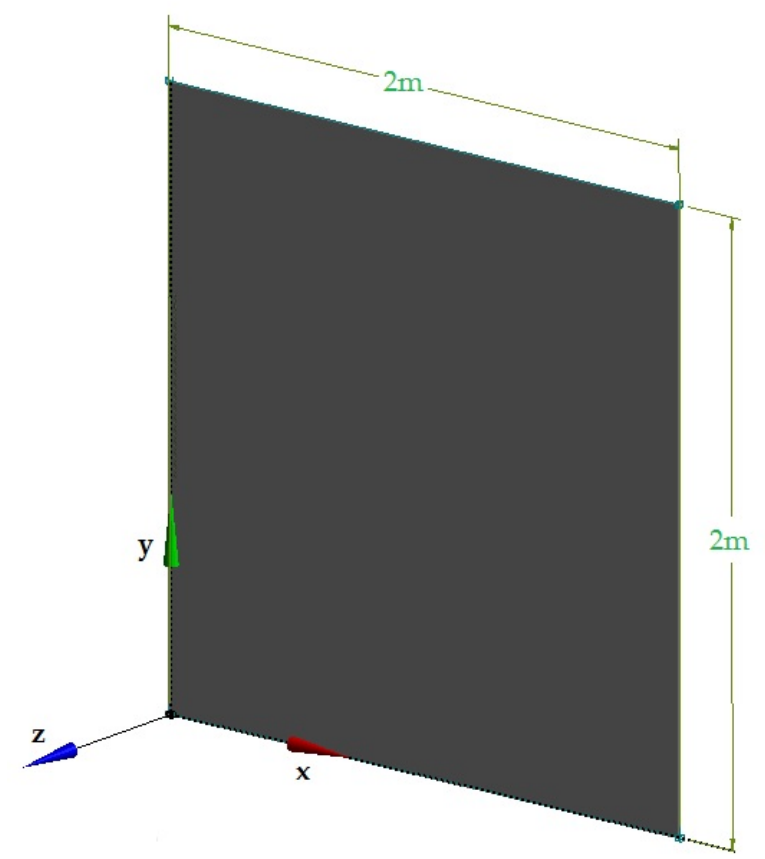

Figure 1: Flat aluminum 6061 target, dimensions $2000 \times 2000 \times 5 \mathrm{~mm}$.

Equations 6 and 7 have been developed for a uniform grid, while equation 8 for a non-uniform one. A nonuniform grid denser near the boundary surfaces was selected because high sensitivity was observed in the discretization of the target's depth. Equations 6, 7 and 8 are expressions of second order accuracy $O\left(\Delta^{2}\right)$.

For time marching the time derivative is numerically approximated as

$$
\frac{d T}{d t}=\frac{T^{n+1}-T^{n}}{\Delta t}
$$

and applying the Crank-Nicolson scheme [13] (combination of forward Euler explicit scheme and backward Euler implicit scheme) we conclude to a sparse linear system of equations with seven non-zero diagonals. For the closure of the system of equations appropriate boundary conditions are implemented (equation 10) to take into account convection and radiation losses in the boundary nodes.

$$
k_{t} \cdot \frac{d T}{d n}=q_{s}-h_{c o n} \cdot\left(T-T_{\infty}\right)-\sigma \cdot \epsilon \cdot\left(T^{4}-T_{\infty}^{4}\right)
$$

$\mathrm{n}$ indicates the normal direction, $q_{s}$ is the constant heat flux because of sunlight reflection on the target, $h_{c o n}$ is the heat transfer coefficient expressing convection losses, $\sigma$ is the Stefan-Boltzmann constant and $\epsilon$ is the emissivity of the target which is assumed to be constant.

While Crank-Nicolson scheme showed satisfactory stability for large time steps, solution dependence on the time step was observed necessitating the fulfillment of the CFL condition [14] given in equation 11 .

$$
\frac{k_{t}}{\rho \cdot C_{p}} \cdot\left(\frac{\Delta t}{\Delta x^{2}}+\frac{\Delta t}{\Delta y^{2}}+\frac{\Delta t}{\Delta z^{2}}\right) \leq \frac{1}{2}
$$

\section{Results}

\subsection{Parametric Analysis}

The numerical model which has been presented above was applied for testing how the parameters " $h$ " heat transfer coefficient and " $\epsilon$ " target's emissivity affect the applicability of the methodology. We assume that we have an aluminum 6061 target $2000 \times 2000 \times 5 \mathrm{~mm}$ (figure 1 ) of $54 \mathrm{~kg}$, with a thermal conductivity equal to $167 \mathrm{~W} / \mathrm{m} / \mathrm{K}$ and a specific heat capacity at constant pressure equal to $900 \mathrm{~J} / \mathrm{kg} / \mathrm{K}$. These properties correspond to the $10^{\circ}$ inclined target placed in PROTEAS facilities for which experimental data will be given in Section 4.2 sub-section.

Five cases were solved to test the applicability of the methodology for different heat transfer coefficients and emissivity constants. In case 1 we assume that we have a constant heat transfer coefficient corresponding to the natural convection case of a $10^{\circ}$ inclined plate with the heated surface being the lower surface ( [15], [16], [17]), equal to $5.6 \mathrm{~W} / \mathrm{m}^{2} / \mathrm{K}$ and an emissivity equal to 0.5 . Then in cases 2,3 and 4 the heat transfer coefficient is $15 \mathrm{~W} / \mathrm{m}^{2} / \mathrm{K}$ and the target emissivity is equal to $0,0.5$ and 1 respectively. In case 5 the heat transfer coefficient is $25 \mathrm{~W} / \mathrm{m}^{2} / \mathrm{K}$ and the target emissivity is equal to 0.5 . Heat transfer coefficents equal to $15 \mathrm{~W} / \mathrm{m}^{2} / \mathrm{K}$ and $25 \mathrm{~W} / \mathrm{m}^{2} / \mathrm{K}$ correspond to typical forced convection cases [15]. In all the above mentioned cases we assume that only one heliostat focuses on the target, producing a Gaussian heat flux 

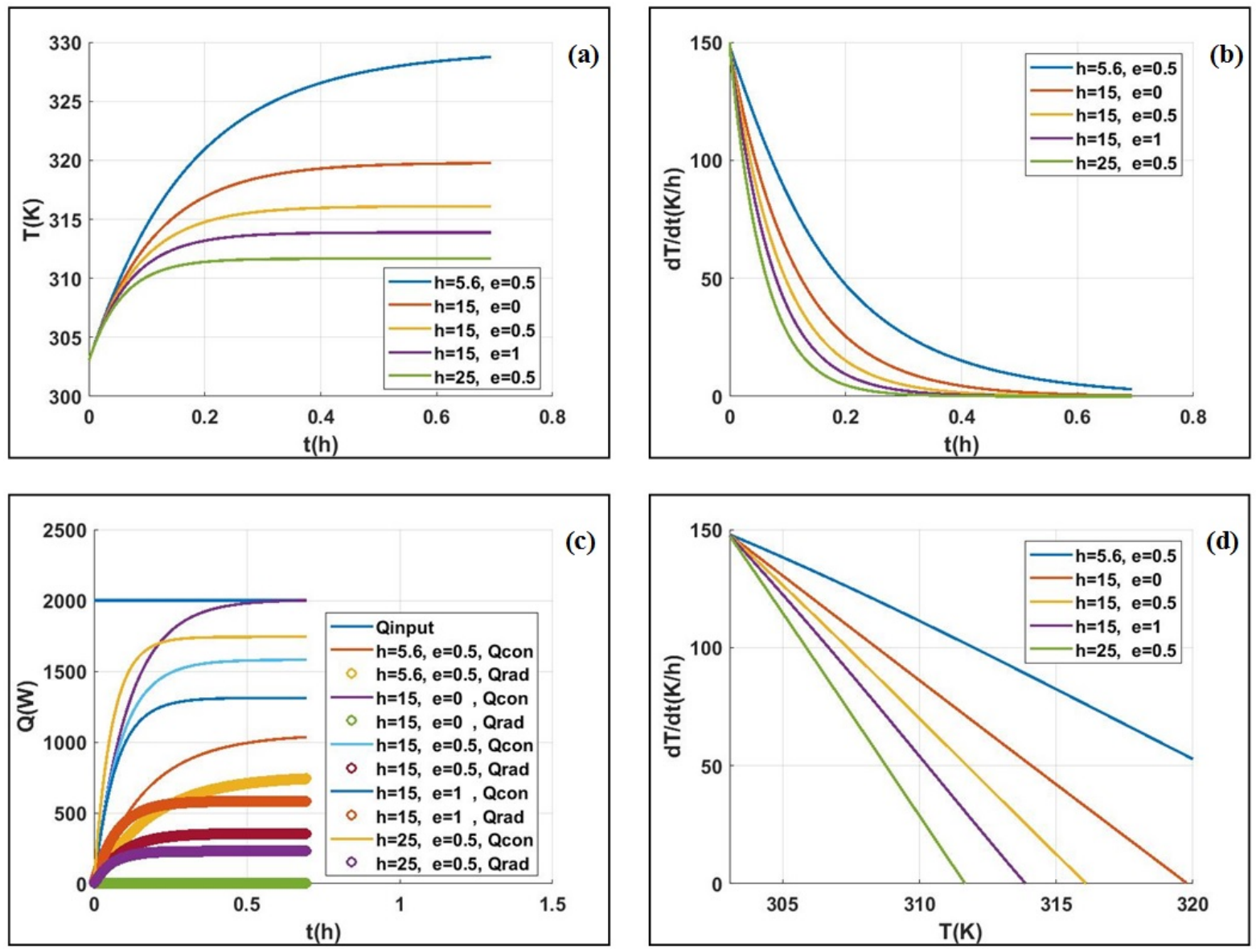

Figure 2: Average temperature evolution over time (a), average temperature time derivative over time (b), heat input power, convective and radiative losses (c), average temperature time derivative versus average temperature $(d)$.

profile on the down facing surface given by 12). Gaussian heat flux profile was selected as it describes quite accurately the distribution when heliostats focus on a target [18], [19].

$q(x, y)=q_{\max } \cdot \exp \left(-\left(\frac{\left(x-x_{0}\right)^{2}}{2 \cdot \sigma_{x}^{2}}+\frac{\left(y-y_{0}\right)^{2}}{2 \cdot \sigma_{y}^{2}}\right)\right)$

The total power is equal to

$$
Q=\int_{0}^{2} \int_{0}^{2} q(x, y) d x d y=2 \cdot \pi \cdot q_{\max } \cdot \sigma_{x} \cdot \sigma_{y}
$$

where $x_{0}=y_{0}=1 \mathrm{~m}$ is the center of the target, $\sigma_{x}$ and $\sigma_{y}$ are assumed to be equal to 0.2 and the total absorbed power for each heliostat is assumed to be equal to $2 \mathrm{~kW}$.

Firstly, a grid dependence study was performed to find the appropriate numerical grid which will give us an independent solution. Testing the weighted average temperature and its time derivative, we concluded that a $21 \times 21 \times 5$ grid, dense in the $z$-direction near the large surfaces is sufficient. The time step which was used and fulfills the CFL condition is $5 \cdot 10^{-4} s$. The initial target temperature and the ambient temperature were equal to $303 \mathrm{~K}$.

In figure 2 the weighted average temperature developed on the surface of the target is presented. In the same figure the time derivative, the convective and radiative losses and the time derivative as a function of the temperature are shown. In figures $2 a, 2 b$ we see that the larger the heat transfer coefficient and the emissivity are the faster the steady state condition is reached. Moreover we see that the temperature increases almost exponentially and the time derivative decreases almost exponentially over time. In figure $2 c$ the input power and the convective and radiative heat losses for all the cases are given. As expected the losses increase over time along with the temperature till the steady state. The convective and radiative heat losses are expressed by equations 14 :

$Q_{c o n}=h_{c o n} \cdot A \cdot\left(T-T_{a m b}\right), Q_{r a d}=\sigma \cdot \epsilon \cdot A \cdot\left(T^{4}-T_{a m b}^{4}\right)$ 
Table 1: Results of linear fitting in several time periods for the fourth test case where $\mathrm{h}=15 \mathrm{~W} / \mathrm{m}^{2} / \mathrm{K}, \epsilon=1$.

\begin{tabular}{cccccccr}
\hline $\begin{array}{c}\text { Test Case } 4 \\
\mathrm{~h}=15 \mathrm{~W} / \mathrm{m}^{2} / \mathrm{K}, \\
\epsilon=1\end{array}$ & $\begin{array}{c}\alpha \\
(1 / h)\end{array}$ & $\begin{array}{c}h_{\text {tot }} \\
{\left[W / \mathrm{m}^{2} / K\right]}\end{array}$ & $\begin{array}{c}h_{\text {con }} \\
{\left[W / \mathrm{m}^{2} / K\right]}\end{array}$ & $\begin{array}{c}h_{\text {rad }} \\
{\left[W / \mathrm{m}^{2} / K\right]}\end{array}$ & $\begin{array}{c}h_{\text {con }}+h_{\text {rad }} \\
{\left[W / \mathrm{m}^{2} / K\right]}\end{array}$ & $\begin{array}{c}\delta h_{\text {tot }} \\
{\left[W / \mathrm{m}^{2} / K\right]}\end{array}$ & $\begin{array}{c}d T / d t \\
(K / h)\end{array}$ \\
\hline $0.7 \mathrm{~h}$ & 13.81 & 23.19 & 15 & 6.62 & 21.62 & 1.57 & 150.26 \\
\hline $100 \mathrm{~s}$ & 13.11 & 22.02 & 15 & 6.37 & 21.37 & 0.65 & 148.48 \\
\hline $50 \mathrm{~s}$ & 12.90 & 21.65 & 15 & 6.34 & 21.34 & 0.31 & 148.27 \\
\hline $10 \mathrm{~s}$ & 12.69 & 21.30 & 15 & 6.32 & 21.32 & -0.02 & 148.19 \\
\hline $5 \mathrm{~s}$ & 12.66 & 21.25 & 15 & 6.31 & 21.31 & -0.07 & 148.19 \\
\hline $2.5 \mathrm{~s}$ & 12.64 & 21.23 & 15 & 6.31 & 21.31 & -0.08 & 148.19 \\
\hline $1 \mathrm{~s}$ & 12.64 & 21.23 & 15 & 6.31 & 21.31 & -0.08 & 148.19 \\
\hline
\end{tabular}

When the emissivity factor is equal to 0 , only convective heat losses exist. In all other cases the radiative losses can be linearized for low temperatures [15] and in such a case they are expressed according to equation 15:

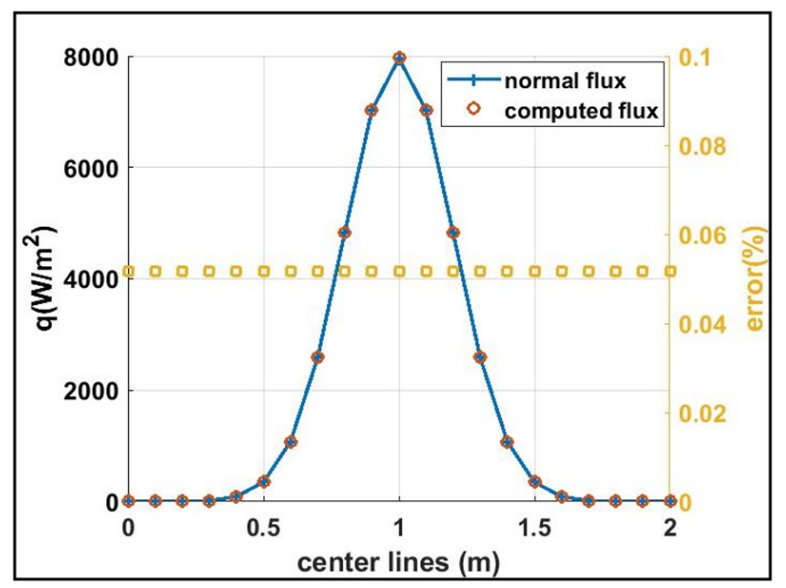

Figure 3: Comparison of the normally distributed and computed heat fluxes in the center lines for case 4.

$$
Q_{\text {rad }}=h_{r a d} \cdot A \cdot\left(T-T_{a m b}\right)
$$

where

$$
h_{r a d}=\epsilon \cdot \sigma \cdot\left(T+T_{a m b}\right) \cdot\left(T^{2}+T_{a m b}^{2}\right)
$$

Additionally, in figure $2 \mathrm{~d}$ we see that the time derivative as a function of temperature is almost linear for all the cases. It is the radiative losses which introduce the non-linearity when the temperature increases. Assuming all these curves to be linear with a quite good accuracy, the total heat transfer coefficient is given by equation 17 .

$$
h_{t o t}=\frac{a \cdot m \cdot C_{p}}{A}
$$

where $a$ is the slope of the linear equation.

What is important and constitutes the basis of the presented methodology is the fact that in all the cases (regardless of the heat transfer coefficient and the emissivity) the time derivative in the beginning of the simulations (in the ambient temperature) is the same. If we multiply this time derivative with the mass and the specific heat capacity of the target we have the total power which was set as a boundary condition, i.e. $2 \mathrm{~kW}$.

Case $4\left(\mathrm{~h}=15 \mathrm{~W} / \mathrm{m}^{2} / \mathrm{K}, \epsilon=1\right)$, where the radiative effects are more prominent was further analyzed. Linear fitting was applied for the temperature time derivative as a function of temperature. This fitting was applied for the values from the beginning of the calculated derivatives till a percentage of the whole simulation time. The purpose was to check how the linear fitting is affected by the radiative effects, as radiative effects grow over time. In table 1 for these time periods the slope of the linear fitting, the total heat transfer coefficient from equation 17 , the constant heat transfer coefficient because of convection, the heat transfer coefficient because of radiation (equation 16) for the mean temperature in the investigated time period, the total heat transfer coefficient as a summation of the previous two heat transfer coefficients and its discrepancy with the total coefficient in the third row are given. In the last row the temperature time derivative calculated in the ambient temperature is given according to the linear fitting parameters, applying extrapolation.

In table 1 we see that for shorter time periods the linear fitting slope decreases till $12.64(1 / \mathrm{h})$. The same behavior is observed for the total heat transfer coefficient and the heat transfer coefficient because of radiation. They converge to $21.23\left(\mathrm{~W} / \mathrm{m}^{2} / \mathrm{K}\right)$ and $6.31\left(\mathrm{~W} / \mathrm{m}^{2} / \mathrm{K}\right)$ respectively. The absolute value of the difference between the total heat transfer coeffi- 
Table 2: $x-y$ coordinates for each thermocouple placed on the rear side of the target.

\begin{tabular}{cccccccccccc}
\hline TC\# & $x(\mathrm{~m})$ & $\mathrm{y}(\mathrm{m})$ & TC\# & $\mathrm{x}(\mathrm{m})$ & $\mathrm{y}(\mathrm{m})$ & TC\# & $\mathrm{x}(\mathrm{m})$ & $\mathrm{y}(\mathrm{m})$ & TC\# & $\mathrm{x}(\mathrm{m})$ & $\mathrm{y}(\mathrm{m})$ \\
\hline 1 & 1.0 & 1.0 & 11 & 1.0 & 1.2 & 21 & 1.4 & 1.0 & 31 & 1.8 & 1.4 \\
\hline 2 & 0.9 & 1.1 & 12 & 1.2 & 1.2 & 22 & 1.4 & 0.6 & 32 & 1.8 & 1 \\
\hline 3 & 1.0 & 1.1 & 13 & 1.2 & 1.0 & 23 & 1.0 & 0.6 & 33 & 1.8 & 0.6 \\
\hline 4 & 1.1 & 1.1 & 14 & 1.2 & 0.8 & 24 & 0.6 & 0.6 & 34 & 1.8 & 0.2 \\
\hline 5 & 1.1 & 1.0 & 15 & 1.0 & 0.8 & 25 & 0.6 & 1.0 & 35 & 1.4 & 0.2 \\
\hline 6 & 1.1 & 0.9 & 16 & 0.8 & 0.8 & 26 & 0.2 & 1.8 & 36 & 1.0 & 0.2 \\
\hline 7 & 1.0 & 0.9 & 17 & 0.8 & 1.0 & 27 & 0.6 & 1.8 & 37 & 0.6 & 0.2 \\
\hline 8 & 0.9 & 0.9 & 18 & 0.6 & 1.4 & 28 & 1.0 & 1.8 & 38 & 0.2 & 0.2 \\
\hline 9 & 0.9 & 1.0 & 19 & 1.0 & 1.4 & 29 & 1.4 & 1.8 & 39 & 0.2 & 0.6 \\
\hline 10 & 0.8 & 1.2 & 20 & 1.4 & 1.4 & 30 & 1.8 & 1.8 & 40 & 0.2 & 1.0 \\
\hline & & & & & & & & & 41 & 0.2 & 1.4 \\
\hline
\end{tabular}

cients also decreases till the 10 s time period and then slightly increases to a steady value. Finally, we also observe that the temperature time derivative reach the value of $148.19(\mathrm{~K} / \mathrm{h})$. Multiplying this value with the mass specific heat capacity of the target (equation 18) we see that the total sun power is calculated with a $0.03 \%$ accuracy.

$$
P_{t o t}=m \cdot C_{p} \cdot \frac{d T}{d t}=2000.565 \mathrm{~W}
$$

Here it should be noted that although for shorter time periods the time derivative is closer to the value which will give us the expected power, for longer time periods the time derivative is not far from the desired one. For example for the $0.7 \mathrm{~h}$ period the total power is calculated to be $2028.51 \mathrm{~W}$ which has a $1.43 \%$ difference from the set total power. The described converging behavior is also observed in the other cases.

The same approach could be applied for each node separately, multiplying this time with the mass and dividing with the area which correspond to each node to find the heat flux on the target surface. In figure 3 the calculated heat flux for case 4 is compared to the normal distribution which was set as a boundary condition, along with the absolute error in the center $x$ and $y$ lines. We see that the similarity is quite good.

\subsection{Experimental Application}

The above mentioned methodology for the measurement of the heat flux and the total power was experimentally tested on the target above the iSTORE receiver at PROTEAS facilities. In its rear side 41 Class-1 k-type thermocouples operating till $260^{\circ} \mathrm{C}$ are welded for monitoring the developed temperatures [20]. The position of the thermocouples ac- cording to the reference system in figure 4 are given in table 2.

Several experiments on the target above iSTORE receiver have been conducted to test the applicability of the presented methodology. Below such a typical experiment will be presented. One and then two heliostats aimed the target and then they were removed to cool it down. In figure 4 the heat flux distribution is shown after triangulation-based cubic interpolation [21] for one heliostat and both heliostats aiming on the target. Integrating in space the heat flux distribution the total heat power absorbed by the target was calculated to be $879 \mathrm{~W}$ in the first phase and $1678 \mathrm{~W}$ in the second phase. These values are much less than what a heliostat can produce because of the low absorptivity of the white painted target.

In figure 5 the average temperature over time and the average temperature time derivative as a function of time and temperature are given for the three phases. In the first phase of the experiment we see that when the heliostat aims the target the temperature augments till steady state, where the temperature derivative becomes zero. Then adding the second heliostat the temperature raises further and in the cooling phase the temperature decreases till the ambient temperature. We also see that linear fitting is presented for the whole period of each phase. We see that these lines in the three phases are almost parallel, as were expected. 

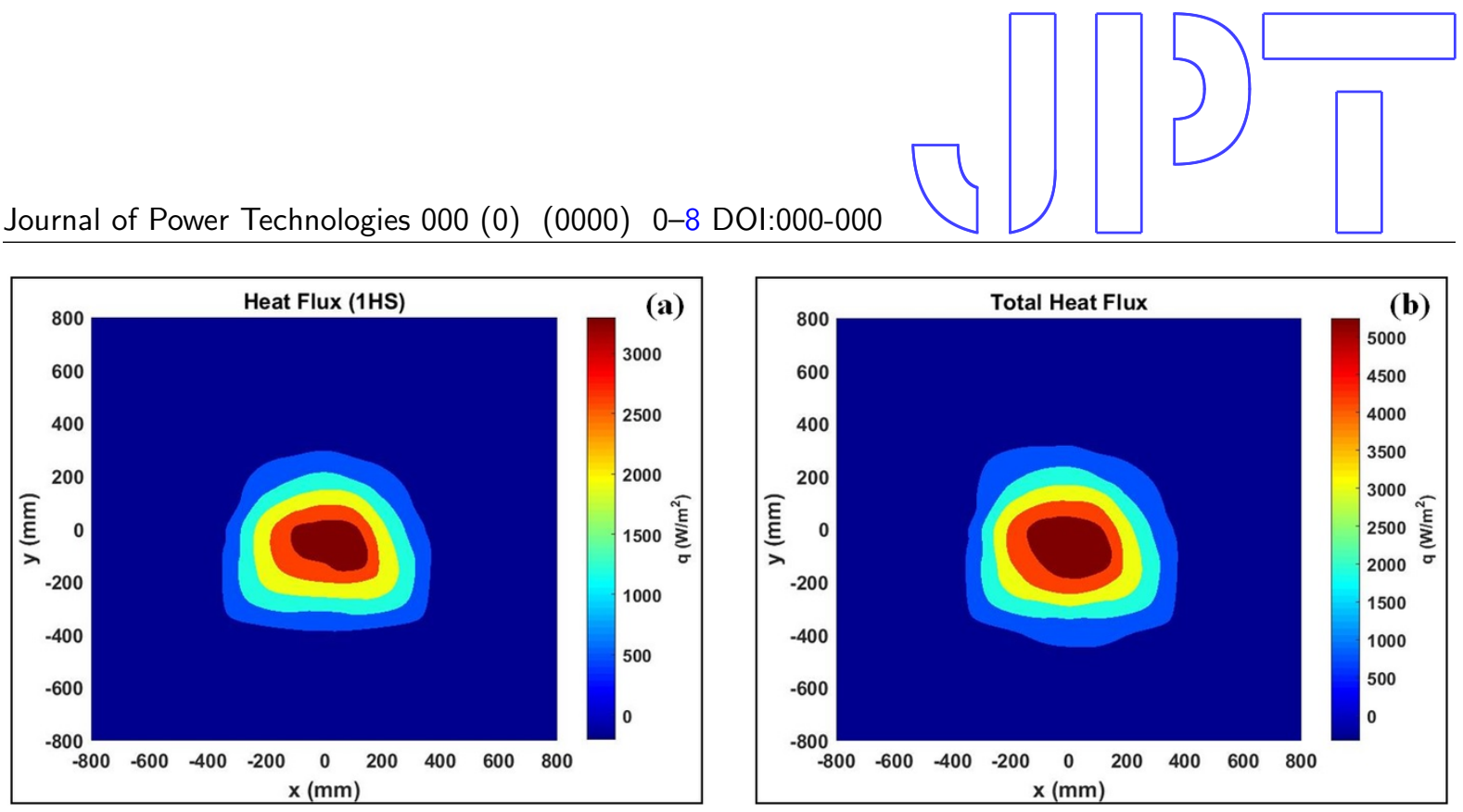

Figure 4: Experimental results. Heat flux distribution for 1 healiostat aiming on the target (a), total heat flux distribution for 2 heliostats aiming on the target (b).
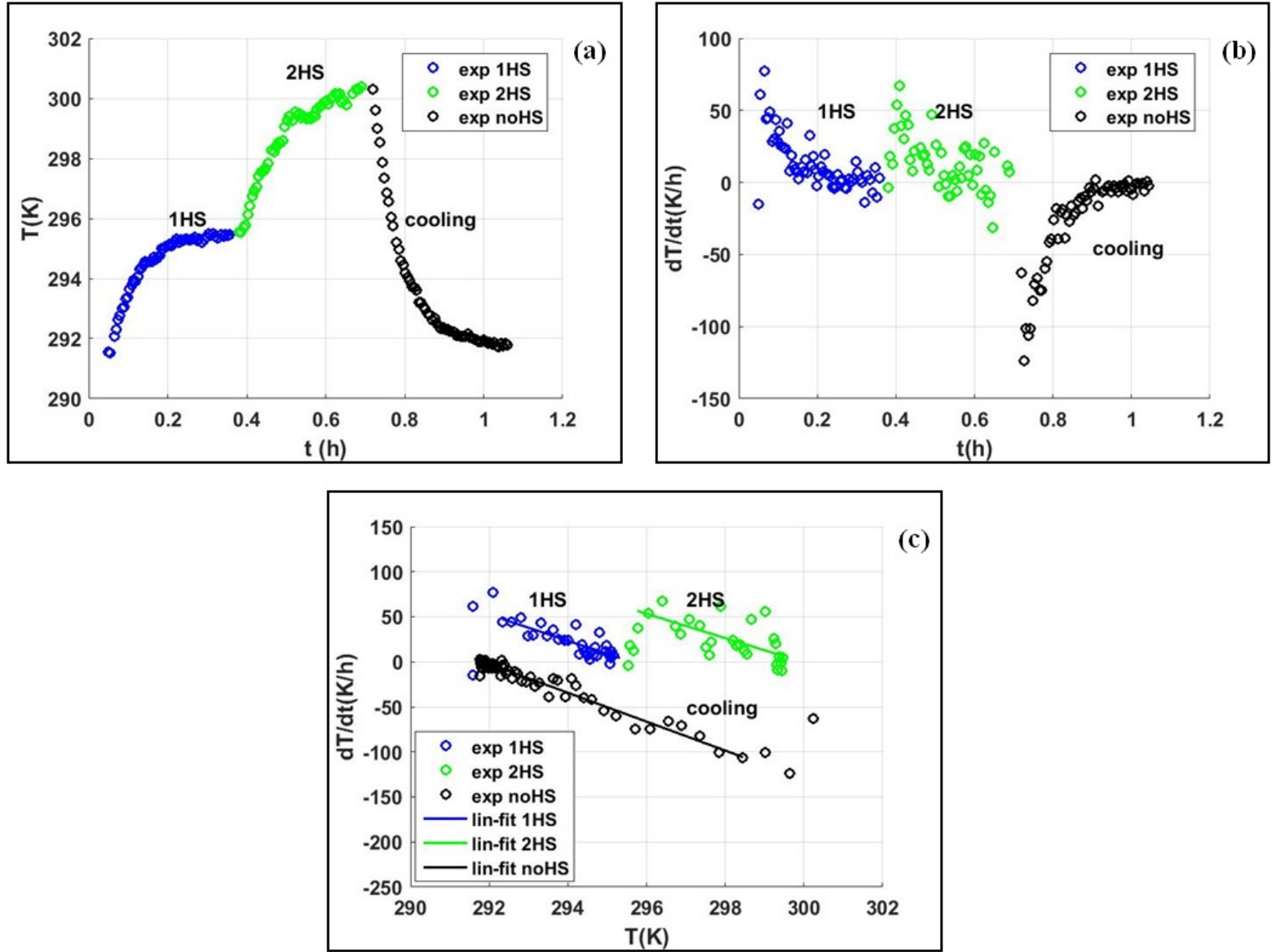

Figure 5: Experimental results. Average temperature evolution over time (a), average temperature time derivative (b), average temperature time derivative versus average temperature (c).

\section{Summary/Conclusions}

In this paper the concept of a new methodology for the flux measurement in CSP applications was presented and applied experimentally on a flat target, though it can be also applied to either external or internal receivers. It is a methodology which could be categorized in the direct methods. The accurate knowledge of the properties of the target material and the target 
geometry is necessary. Then, with appropriate analysis of the temperature evolution on the target, heat flux distribution is calculated. Having the heat flux distribution, the heat input power is calculated with its spatial integration as in the well known direct approaches.

\section{Acknowledgements}

The creation of the PROTEAS Field Facility, where the application of the proposed methodology took place, was made possible through the STEP-EW project, which was cofunded by the European Regional Development Fund through the European Territorial Cooperation Programme Greece-Cyprus 20072013. We would like to thank the Joint Technical Secretariat of this Programme, as well as our project partners in the project, the Electricity Authority of Cyprus, the Water Development Department, and the Foundation for Research and Technology Hellas (Diktyo Praxi).

The research leading to these results has also received funding from the European Union Seventh Framework Programme (FP7/2007-2013) under grant agreement number 609837 and from the Horizon 2020 Programme under the INSHIP project with grant agreement number 731287 .

\section{References}

1.Roger, M., Herrmann, P., Ulmer, S., Ebert, M., Prahl, C., Gohring, F. (2014) Techniques to measure solar flux density distribution on large-scale receivers. Journal of Solar Energy Engineering , 126 , 031013.

2.King, D.L., Arvizu, D.E. (1981) Heliostat characterization at the central receiver test facility. Journal of Solar Energy Engineering , 103 , 82-88.

3.Kodama, T., Gokon, N., Matsubara, K., Yoshida, K., Koikari, S., Yagase, Y., Nakamura, K. (2014) Flux measurement of a new beam-down solar concentrating system in Miyazaki for demonstration of thermochemical water splitting reactors. Energy Procedia , 49 , 1990-1998.

4.Bellestrin, J., Estrada, C.A., Rodriguez-Alonso, M., Perez-Rabago, C., Langley, L.W., Barnes, A. (2004) High-heat-flux sensor calibration using calorimetry. Metrologia , 41 , 314-318.

5.Bellestrin, J., Estrada, C.A., Rodriguez-Alonso, M., Perez-Rabago, C., Langley, L.W., Barnes, A. (2006) Heat flux sensors: Calorimeters or radiometers?. Solar Energy , 80 , 1314-1320.
6.Guillot, E., Alxneit, I., Ballestrin, J., Sans, J.L., Willsh, C. (2014) Comparison of 3 heat flux gauges and a water calorimeter for concentrated solar irradiance measurement. Energy Procedia , 49 , 20902099.

7.Ulmer, S., Reinalter, W., Heller, P., Lupfert, E., Martinez, D. (2002) Beam Characterization and improvement with a flux mapping system for dish concentrators. Journal of Solar Energy Engineering , 124 , 182-188.

8.Ho, C.K., Khalsa, S.S. (2012) A photographic flux mapping method for concentrating solar collectors and receivers. Journal of Solar Energy Engineering , 134 , 041004.

9.Lee, H., Chai, K., Kim, J., Lee, S., Yoon, H., Yu, C., Kang, Y. (2014) Optical perforamnce evaluation of a solar furnace by measuring the higly concentrated solar flux. Energy , 66 , 63-69.

10.Ferriere, A., Volut, M., Perez, A., Volut, Y. (2015) In-situ measurement of concentrated solar flux and distribution at the aperture of a central solar receiver. AIP Conf. Proc. 1734, SolarPACES , 130007-1130007-8.

11.Ebert, M., Benitez, D., Roger, M., Korzynietz, R., Brioso, J.A. (2016) Efficiency determination of tubular solar receivers in central receiver systems. Solar Energy , 139 ,179-189.

12.Papanicolas, C.N., Bonanos, A.M., Georgiou, M.C., Gulliet, E., Jarraud, N., Marakkos, C., Montenon, A., Stiliaris, E., Tsioli, E., Tzamtzis, G., Votyakov, E.V. (2016) CSP cogeneration of Electricity and Desalinated Water at the Pentakomo Field Facility. AIP Conf. Proc. 1734, SolarPACES , 100008.

13. Hirsch, C. (1988) Numerical computation of internal and external flows. John Wiley \& Sons, Vol. 1.

14.Courant, R., Friedrichs, K., Lewy, H. Über die partiellen Differenzengleichungen der mathematischen Physik. Mathematische Annalen , 100 , 32-74.

15.Cengel, Y.A. (2003) Heat transfer - A practical approach. McGraw Hill, second edition.

16.Churchill, S.W., Chu, H.H.S. (1975) Correlating Equations for Laminar and Turbulent free convection from a Vertical plate. International Journal of Heat Mass Transfer , 1823.

17.Fujii, T., Imura, H. (1972) Natural Convection Heat Transfer from a plate in arbitrary inclination. International Journal of Heat Mass Transfer , 15 ,755.

18.Schwarzbolz, P., Schmitz, M., Pitz-Paal, R. (2009) Visual HFCAL - a software for layout and optimization 
of heliostat fields. SolarPACES , Berlin, September 15-18.

19.Collado, F.J. (2010) One-point fitting of the flux density produced by a heliostat. Solar Energy , 84 ,673-684.

20.Stokos, K.G., Stiliaris, E., Bonanos, A.M., Georgiou, M.C., Guillen, E., Montenon, A., Papanicolas, C.N. (2018) The Control System at PROTEAS. AIP Conference Proceedings 210019.

21. Matlab R2016a, The Mathworks, Inc., Natick, Massachusetts, United States. 